\title{
EsCALA DE ESFORÇO PERCEBIDO NA FLEXIBILIDADE (PERFLEX): UM INSTRUMENTO ADIMENSIONAL PARA SE AVALIAR A INTENSIDADE?
}

Estélio Henrique Martin Dantas'estelio@cobrase.org.br Pablo Teixeira Salomão² pablomestrado@yahoo.com.br Rodrigo Gomes de Souza Vale ${ }^{2,4}$ rodrigovale@globo.com

\author{
Abdallah Achour Júnior ${ }^{5}$ achour@onda.com.br \\ RobertoSimão6robertosimao@superig.com.br \\ Nébia Maria Almeida de Figueiredo7 nebia@unirio.br
}

doi:10.3900/fpi.7.5.289.p

Dantas EHM, Salomão PT, Vale RGS, Achour Júnior A, Simão R, Figueiredo NMA. Escala de esforço percebido na flexibilidade (PERFLEX): um instrumento adimensional para se avaliar a intensidade? Fit Perf J. 2008 set-out; 7(5):289-94.

\section{RESUMO}

Introdução: A dificuldade de se contar com instrumentos de avaliação capazes de avaliar o estresse físico laboral, decorrente da realização de arcos de movimentos de amplitude máxima nas atividades de grupos profissionais, principalmente na área de saúde (enfermeiros, fisioterapeutas e médicos, em especial), foi o fator motivador preponderante para a execução da pesquisa. $\bigcirc$ objetivo deste estudo é buscar a correlação porventura existente entre a intensidade e a amplitude de arcos de movimentos articulares (avaliados por meio da Escala de Esforço Percebido na Flexibilidade - PERFLEX) com um método objetivo de avaliação da flexibilidade. Materiais e Métodos: A amostra foi composta por um grupo de 42 alunos voluntários, de ambos os sexos, da Escola Superior de Educação Física de Muzambinho, Minas Gerais, nãoatletas, variando entre 19 anos e 27 anos. A PERFLEX possui cinco níveis de intensidades, variando de 0 a 110 , categorizados em cinco descritores verbais, para que o avaliando possa discernir, através da descrição da sua percepção, qual a sensação correspondente à amplitude de movimento realizado: de 0 a 30 - "Normalidade"; de 31 a 60 - "Forçamento"; de 61 a 80 - "Desconforto"; de 81 a 90 - "Dor suportável"; e de 91 a 110 - "Dor forte". Paralelamente à avaliação da percepção subjetiva de esforço, durante a realização do movimento ao longo de todo o arco articular, se mensurava o ângulo do mesmo, por meio de um goniômetro, e a força aplicada para se conseguir o citado arco, por meio de um dinamômetro. A goniometria foi feita segundo o protocolo LABIFIE. Resultados: Os resultados das correlações estatisticamente significativas (para $\mathrm{p}<0,05$ ) entre as médias angulares e as de tensão aplicada no segmento corporal, para os níveis da PERFLEX, foram: na categoria "Mobilidade" (índices de 31 a 60), observou-se correlação nos movimentos de extensão horizontal do ombro $(r=0,62 ; p=0,029)$ e de abdução de membros inferiores $(r=0,63 ; p=0,025)$; na categoria "Alongamento" (índices de 61 a 80), a correlação pode ser observada também na extensão horizontal do ombro ( $r=0,76$; $p=0,004)$. Discussão: $\bigcirc$ estudo indica que a PERFLEX pode vir a se constituir num instrumento válido, de avaliação do nível da intensidade exigida para a realização de um movimento de amplitude angular máxima.

\section{PALAVRAS-CHAVE}

Exercícios de Alongamento Muscular, Amplitude de Movimento Articular, Goniometria Articular.

\footnotetext{
1 Universidade Castelo Branco - UCB - Programa de Pós-graduação Stricto Sensu em Ciência da Motricidade Humana - PROCIMH - Rio de Janeiro - Brasil

${ }^{2}$ Universidade Castelo Branco - UCB - Laboratório de Biociências da Motricidade Humana - LABIMH - Rio de Janeiro - Brasil

${ }^{3}$ Bolsista de produtividade em Pesquisa - CNPq - Brasil

${ }^{4}$ Universidade Federal do Rio Grande do Norte - UFRN - Programa de Pós-Graduação em Ciências da Saúde - PPGCSa - Natal - Brasil

${ }^{5}$ Universidade Estadual de Londrina - Londrina - Brasil

${ }^{6}$ Universidade Federal do Rio de Janeiro - Rio de Janeiro - Brasil

7 Universidade Federal do Estado do Rio de Janeiro - UNIRIO - Rio de Janeiro - Brasil
}

Copyright(C 2008 por Colégio Brasileiro de Atividade Física, Saúde e Esporte

Fit Perf J | Rio de Janeiro | 7 | 5 | 289-294 | set/out 2008 


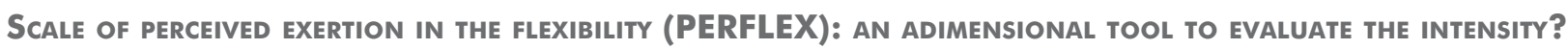
ABSTRACT

Introduction: The difficulty of counting with evaluation tools capable to evaluate the labor physical estresse, due to the accomplishing of movements arches of maximum amplitude in the activities of professional groups, mainly in the health area (nurses, physiotherapists and physicians, especially) was the preponderant motivator factor for the research execution. The objective of this study is to search the correlation by chance existent between the intensity and the amplitude of articular movement arches (evaluated through the Scale of Noticed Effort in the Flexibility - PERFLEX) - with an objective method of evaluation of the flexibility. Materials and Methods: The sample was composed by a group of 42 voluntary students, of both sexes, of the Superior School of Physical Education of Muzambinho (Escola Superior de Educação Física de Muzambinho), Minas Gerais, non-athletes, varying between 19 and 27 years. PERFLEX possesses five intensities levels, varying from 0 to 110 , classified in five verbal descriptors, so that the evaluator_can discern it, through the description of its perception, which the sensation corresponding to the amplitude of accomplished movement: from 0 to 30 - "Normality"; from 31 to 60 - "Forcing"; from 61 to 80 - "Discomfort"; from 81 to 90 - "Bearable Pain"; and from 91 to 110 - "Strong Pain." Parallel to the evaluation of the subjective effort perception, during the accomplishing of the movement along the whole articular arch, was measured the angle of the same (through a goniometer) and the applied force to get the quoted arch (through a dynamometer). The goniometry was made, following the LABIFIE protocol. Results: The results of the statistically significant correlations (for $p<0.05$ ) between angular average (of the goniometry) and the applied tension in the body segment (dynamometry), for the levels of PERFLEX were: in the "Mobility" category, (indexes from 31 to 60) correlation was observed in the movements of Horizontal Shoulder Extension ( $r=0.62 ; p=0.029)$ and of Inferior Limbs Abduction ( $r=0.63 ; p=0.025)$; in the "Stretching" category, (indexes from 61 to $80)$ the correlation can be observed, also in the Horizontal Shoulder Extension ( $r=0.76 ; p=0.004)$. Discussion: The study indicates that PERFLEX can come to turn in a valid instrument, of evaluation of the intensity level demanded for the accomplishing of a movement of maximum angular amplitude.

\section{KEYWORDS}

Muscle Stretching Exercises, Range of Motion, Articular, Goniometry, Articular.

\section{Escala de esfuerzo percibido en la flexibilidad (Perflex): ¿un instrumento adimensional para eValuarse la INTENSIDAD?}

\section{RESUMEN}

Introducción: La dificultad de contarse con instrumentos de evaluación capaces de evaluar el estrés físico laborable, decurrente de la realización de arcos de movimientos de amplitud máxima en las actividades de grupos profesionales, sobre todo en el área de salud (enfermeros, fisioterapeutas y médicos, en especial) fue el factor motivador preponderante para la ejecución de la investigación. Objetivo: El objetivo de este estudio es buscar la correlación quizá existente entre la intensidad y la amplitud de arcos de movimientos articulares (evaluados por medio de la Escala de Esfuerzo Percibido en la Flexibilidad - PERFLEX) - con un método objetivo de evaluación de la flexibilidad. Materiales y Métodos: La muestra fue compuesta por un grupo de 42 alumnos voluntarios, de ambos los sexos, de la Escuela Superior de Educación Física de Muzambinho, Minas Gerais, no atletas, variando entre 19 y 27 años. La PERFLEX posee cinco niveles de intensidades, variando de 0 a 110, categorizados en cinco descriptores verbales, para que lo evaluando pueda discernir, a través de la descripción de su percepción, cual la sensación correspondiente a la amplitud de movimiento realizado: de 0 a 30 - "Normalidad"; de 31 a 60 - "Forzado"; de 61 a 80 - "Incomodidad"; de 81 a 90 - "Dolor soportable"; y de 91 a 110 - "Dolor fuerte". Paralelamente a la evaluación de la percepción subjetiva de esfuerzo, durante la realización del movimiento a lo largo de todo el arco articular, se mensuraba el ángulo del mismo (mediante uno goniómetro) y la fuerza aplicada para conseguirse el citado arco (mediante un dinamómetro). La goniometría fue hecha, siguiendo el protocolo LABIFIE. Resultados: Los resultados de las correlaciones estadísticamente significativas (para $p<0,05$ ) entre las medias angules (de la goniometría) y las de tensión aplicada en el segmento corporal (dinamometría), para los niveles de la PERFLEX fueron: en la categoría "Movilidad" (índices de 31 a 60), se observó correlación en los movimientos de Extensión Horizontal del Hombro ( $r=0,62 ; p=0,029)$ y de Abducción de Miembros Inferiores ( $r=0,63 ; p=0,025)$; en la categoría "Estiramiento" (índices de 61 a 80), la correlación puede ser observada, también en la Extensión Horizontal del Hombro ( $r=0,76 ; p=0,004)$. Discusión: El estudio indica que la PERFLEX puede venir a constituirse en un instrumento válido, de evaluación del nivel de la intensidad exigida para la realización de uno movimiento de amplitud angular máxima.

\section{PALABRAS CLAVE}

Ejercicios de Estiramiento Muscular, Rango del Movimiento Articular, Goniometría Articular.

\section{INTRODUÇÃO}

As atividades cotidianas, laborais e desportivas utilizam, em diversas oportunidades, os arcos máximos do movimento autônomo. Esses arcos de movimento são limitados pelas condições de flexibilidade dos segmentos musculares envolvidos nas respectivas articulações. Portanto, os segmentos musculares necessitam de um bom nível de flexibilidade para que ocorra a utilização completa destes arcos articulares, sem chegar à região de alta resistência ao movimento' ${ }^{1}$.
Na prática laboral, os profissionais da área de saúde, notadamente enfermeiros, fisioterapeutas e médicos, têm a necessidade de realizar movimentos articulares de amplitude máxima, com níveis de estresse físico muito difíceis de serem mensurados pelos métodos formais de avaliação da flexibilidade ${ }^{2}$. A impossibilidade de se usar os métodos lineares ou os angulares na avaliação on the job do estresse físico laboral, constituiu-se num fator motivador importante para o presente estudo. 
nível de flexibilidade está diretamente relacionado à qualidade de vida, sendo necessários valores mínimos de amplitude articular ${ }^{3}$, para possibilitar a realização das atividades da vida diária e laborais dentro dos limites de baixa resistência ao movimento, pois estes não produzem fadiga precoce ${ }^{2}$.

Exercícios de flexibilidade têm sido considerados, por muito tempo, como parte essencial nos procedimentos de aquecimento e indispensável na aptidão física, sendo um método de melhoria da eficiência do movimento, tendo direta relação com o desempenho muscular ${ }^{4,5}$, além de benefícios qualitativos e quantitativos, quer nas performances físicas ${ }^{6}$, quer nas laborais.

A obtenção de um bom nível de flexibilidade através da realização regular de exercícios, sejam eles realizados de forma máxima ou submáxima, influencia positivamente a capacidade motora, impedindo restrições à realização dos arcos de movimento necessários às atividades cotidianas ou laborais ${ }^{\mathbf{7}} \mathbf{8}$.

Embora a Percepção Subjetiva do Esforço (PSE) tenha sido inicialmente desenvolvida para o controle da intensidade de exercícios predominantemente aeróbicos ${ }^{9}$, estudos demonstraram evidências que indicaram a sua validade e a sua reprodutibilidade durante outros tipos de exercícios ${ }^{\mathbf{1 0 , 1 1}}$. Tal esforço está intimamente ligado à intensidade do exercício, pois é resultado da integração de sinais aferentes, provenientes dos sistemas músculoesquelético, cardiovascular e pulmonar?.

A estrutura conceitual para a observação do esforço percebido é derivada de duas linhas convergentes de investigação: a da observação de comportamento e a da percepção visual. Através da integração dos sinais centrais e periféricos, a percepção de esforço realizado é apontada pela classificação numérica e/ou pela expressão verbal; assim, a valoração do esforço percebido representa um poderoso instrumento para monitorar a intensidade do esforço e é freqüentemente usada em testes de esforço progressivo ${ }^{\mathbf{1 2}}$.

Os testes padrões de avaliação da percepção subjetiva do esforço normalmente detectam propriedades variadas, como: (a) o peso de um objeto que seja levantado e/ou transportado; (b) o movimento e os seus sentidos e/ou direção; e (c) as expectativas, modos, e habilidades que são antecedentes ou conseqüentes a um evento ${ }^{13}$.

No caso da amplitude de movimento articular é imprescindível se considerar que, para se alcançar o arco máximo de movimento, será necessário passar por um nível submáximo, até chegar ao nível máximo. As formas de trabalho que exploram estes dois níveis são chamadas na literatura, respectivamente, stretching ou alongamento, e overstretching ou flexionamento ${ }^{2,14,15}$. Um instrumento adimensional de avaliação da percepção subjetiva de esforço, necessariamente terá que contemplar essa categorização.

Sendo assim, o presente estudo tem como objetivo buscar a correlação porventura existente entre a intensi- dade e a amplitude de arcos de movimentos articulares (avaliados por meio da Escala de Esforço Percebido para a Flexibilidade - PERFLEX), com um método objetivo de avaliação da flexibilidade (a goniometria), com controle da força aplicada(por meio da dinamometria).

\section{MATERIAIS E MÉTODOS}

\section{Aprovação do estudo}

estudo foi submetido e aprovado pelo Comitê de Ética em Pesquisa da Universidade Castelo Branco sob $n^{\circ} .0015 / 2008$.

\section{Amostra}

A amostra foi composta por um grupo de 42 alunos voluntários da Escola Superior de Educação Física de Muzambinho, MG, sendo 18 do gênero masculino e 24 do gênero feminino, todos ativos, não-atletas, com idade variando entre 19 anos e 27 anos, que, segundo a Organização Mundial de Saúde ${ }^{16}$, representa a idade adulta.

Os voluntários foram previamente informados a respeito do objetivo e dos procedimentos do respectivo estudo. Posteriormente, foram excluídos os indivíduos que apresentaram histórico de patologias ortopédicas, reumatológicas ou neurológicas e, em seguida, os selecionados assinaram um termo de consentimento livre e esclarecido, concordando com a participação na pesquisa, de acordo com a resolução 196/96 do Conselho Nacional de Saúde.

\section{Procedimentos metodológicos}

Realizados os procedimentos preliminares já descritos, passou-se a mensuração dos movimentos de extensão horizontal do ombro (EHO), abdução da articulação do ombro (AAO), flexão da coluna lombar (FCL), flexão do quadril (FQ) e abdução dos membros inferiores (AMI), através de um teste de esforço progressivo, onde cada

\section{Figura 1 - Manual Muscle Test System}

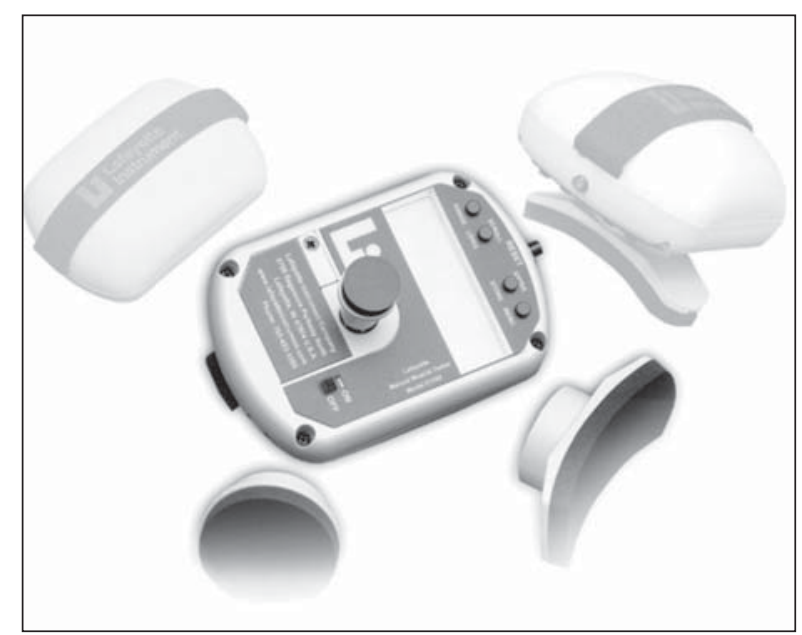


Tabela 1 - Resultados descritivos dos níveis da escala PERFLEX, em função dos resultados da goniometria $\left({ }^{\circ}\right.$ ) e dinamometria (kgf)

\begin{tabular}{|c|c|c|c|c|c|c|c|}
\hline & & \multicolumn{2}{|c|}{$\begin{array}{c}31-60 \\
\text { (mobilidade) }\end{array}$} & \multicolumn{2}{|c|}{$\begin{array}{c}61-80 \\
\text { (alongamento) }\end{array}$} & \multicolumn{2}{|c|}{$\begin{array}{c}81-90 \\
\text { (flexionamento) }\end{array}$} \\
\hline & & média & sd & média & sd & média & sd \\
\hline \multirow{2}{*}{$\mathrm{EHO}$} & dinamometria & 2,3 & 0,8 & 4,2 & 3,1 & 11,0 & 4,8 \\
\hline & goniometria & 52,5 & 8,4 & 78,0 & 15,0 & 105,0 & 16,6 \\
\hline \multirow{2}{*}{$A B D$} & dinamometria & 3,4 & 1,5 & 6,4 & 1,6 & 10,5 & 1,9 \\
\hline & goniometria & 172,5 & 15,7 & 200,0 & 10,8 & 216,5 & 9,8 \\
\hline \multirow{2}{*}{$\mathrm{FCL}$} & dinamometria & 7,4 & 4,6 & 11,4 & 5,6 & 24,9 & 5,3 \\
\hline & goniometria & 13,0 & 11,1 & 23,0 & 12,3 & 29,0 & 14,6 \\
\hline \multirow{2}{*}{$\mathrm{FQ}$} & dinamometria & 4,3 & 1,4 & 5,0 & 1,8 & 8,0 & 2,4 \\
\hline & goniometria & 60,5 & 10,7 & 82,0 & 14,2 & 93,5 & 15,3 \\
\hline \multirow{2}{*}{ AMI } & dinamometria & 2,0 & 1,0 & 3,5 & 1,4 & 8,1 & 2,4 \\
\hline & goniometria & 30,0 & 6,0 & 42,0 & 7,6 & 60,5 & 12,0 \\
\hline
\end{tabular}

sd: desvio padrão; EHO: extensão horizontal do ombro; $A A O$ : abdução da articulação do ombro; $F C L$ : flexão da coluna lombar; FQ: flexão do quadril; e AMI: abdução dos membros inferiores

movimento da flexibilidade é realizado de acordo com o protocolo do LABIFIE de goniometria' ${ }^{\mathbf{1 7}}$.

A avaliação foi realizada utilizando-se, concomitantemente, três instrumentos:

1. Para mensurar a amplitude de movimento foi utilizado um goniômetro de aço (Lafayette, EUA), 14", com escala de $180^{\circ}$ e precisão de $1^{\circ}$.

2. Para quantificar a tensão do esforço, utilizou-se um dinamômetro Manual Muscle Test System - Model 01163 (Lafayette, EUA), com abrangência de zero a $136 \mathrm{kgf}$ e precisão de 0,1 kgf (Figura 1).

3. Para avaliar a percepção do esforço, utilizou-se um método adimensional denominado: Escala de Esforço Percebido na Flexibilidade (PERFLEX), que possui cinco níveis de intensidades, variando de 0 a 110 , categorizados em cinco descritores verbais, para que o avaliando possa discernir, através da descrição da sua percepção, qual a sensação correspondente à amplitude de movimento realizado: de 0 a 30 "normalidade"; de 31 a 60 - "forçamento"; de 61 a 80 - "desconforto"; de 81 a 90 - "dor suportável"; e de 91 a 110 - "dor forte". A PERFLEX apresenta, ainda, uma coluna "Especificação", na qual são descritos os efeitos morfofuncionais dos distintos arcos de movimento no aparelho locomotor, como apresentado em estudos prévios ${ }^{2,18}$ (Figura 2).

A cada sinal do avaliado, de acordo com cada descrição da sensação da escala, o movimento era interrompido e eram coletados e registrados os valores apresentados no goniômetro, no dinamômetro e na PERFLEX (gradação numérica do "nível"). Após a coleta, o movimento era retomado a partir do ponto onde havia parado, até que o avaliado chegasse ao outro nível da escala, quando se repetia o procedimento já descrito. Esse processo continuava até que se chegasse ao limite articular máximo suportado pelo avaliado.

Local, horário e temperatura ambiente para a coleta dos dados foram homogêneos para todos os voluntários. Todas as informações verbais passadas durante a coleta de dados foram padronizadas, para que se evitasse interferência das mesmas nos resultados.

\section{Análise de dados}

O instrumento utilizado para análise estatística foi a versão 14.0 do software SPSS for Windows. Foram utilizadas as seguintes técnicas de estatística descritiva, que possibilitam caracterizar a amostra estudada em função das variáveis selecionadas:

Figura 2 - Escala de Esforço Percebido na Flexibilidade - PERFLEX

\begin{tabular}{|c|c|c|c|}
\hline nível & descrição da sensação & efeito & especificação \\
\hline 30 & normalidade & mobilidade & $\begin{array}{c}\text { não ocorre qualquer tipo de alteração em relação aos } \\
\text { componentes mecânicos, componentes plásticos e compo- } \\
\text { nentes inextensíveis. }\end{array}$ \\
\hline 60 & forçamento & alongamento & $\begin{array}{c}\text { provoca deformação dos componentes plásticos e os com- } \\
\text { ponentes elásticos são estirados ao nível submáximo. }\end{array}$ \\
\hline 80 & desconforto & flexionamento & $\begin{array}{c}\text { provoca adaptações duradouras nos componentes plásti- } \\
\text { cos, elásticos e inextensíveis. }\end{array}$ \\
\hline 110 & dor suportável & possibilidade de lesão & $\begin{array}{c}\text { as estruturas músculo-conjuntivas envolvidas são submeti- } \\
\text { das a um estiramento extremo, causando dor. }\end{array}$ \\
\hline
\end{tabular}


Tabela 2 - Correlação entre os dados obtidos pela goniometria e a dinamometria, em todos os movimentos articulares, de acordo com a PERFLEX

\begin{tabular}{lcccccc}
\hline & \multicolumn{2}{c}{$\begin{array}{c}31-60 \\
\text { (Mobilidade) }\end{array}$} & \multicolumn{2}{c}{$\begin{array}{c}61-80 \\
\text { (Alongamento) }\end{array}$} & \multicolumn{2}{c}{$\begin{array}{c}81-90 \\
\text { (Flexionamento) }\end{array}$} \\
\hline & $\mathrm{r}$ & valor-p & $\mathrm{r}$ & valor-p & $\mathrm{r}$ & valor-p \\
$\mathrm{EHO}$ & $0,62 *$ & 0,029 & $0,76 * *$ & 0,004 & 0,52 & 0,079 \\
$\mathrm{ABD}$ & $-0,12$ & 0,694 & 0,22 & 0,49 & 0,31 & 0,311 \\
$\mathrm{FCL}$ & 0,27 & 0,396 & 0,51 & 0,087 & 0,36 & 0,246 \\
$\mathrm{FQ}$ & $-0,12$ & 0,695 & $-0,03$ & 0,914 & 0,51 & 0,084 \\
$\mathrm{AMI}$ & $0,63 *$ & 0,025 & 0,16 & 0,618 & 0,54 & 0,068 \\
\hline
\end{tabular}

EHO: extensão horizontal do ombro; AAO: abdução da articulação do ombro; FCL: flexão da coluna lombar; FQ: flexão do quadril; e AMl: abdução dos membros inferiores

${ }^{*} p<0,05 ;{ }^{* *} p<0,01$

medidas de tendência central (média) e de dispersão (desvio padrão). A estatística inferencial foi desenvolvida através do teste de Shapiro-Wilk, para verificar a normalidade da amostra do teste de correlação de Pearson, para a verificação do nível de associação entre as variáveis e da análise de variância (ANOVA), seguida do teste post hoc de Tukey.

Como critério de significância estatística, adotou-se o valor de $\mathrm{p}<0,05$.

\section{RESULTADOS}

Optou-se por iniciar a apresentação dos resultados pela estatística descritiva dos dados coletados, conforme apresentado na Tabela 1.

Os valores apresentados na Tabela 1, quando comparados aos obtidos por Silva et al. ${ }^{19}$, revelam que as amplitudes médias encontradas são considerados normais para todos os movimentos avaliados.

Em seguida, como pode ser observado na Tabela 2, buscou-se estabelecer as possíveis correlações existentes entre os dados angulares, obtidos por meio da goniometria, e as mensurações da força aplicada, obtidas pela dinamometria, em cada um dos níveis da PERFLEX.

Destaca-se que, no movimento de extensão horizontal do ombro (EHO), no nível correspondente à "mobilidade", foi encontrada uma correlação classificada como média alta. $\bigcirc$ mesmo acontecendo para o mesmo movimento, no nível correspondente ao "alongamento", para

Gráfico 1 - Múltiplas comparações entre a amplitude dos movimentos articulares, aferidos pelo goniômetro, e as percepções de esforço, conforme a escala PERFLEX

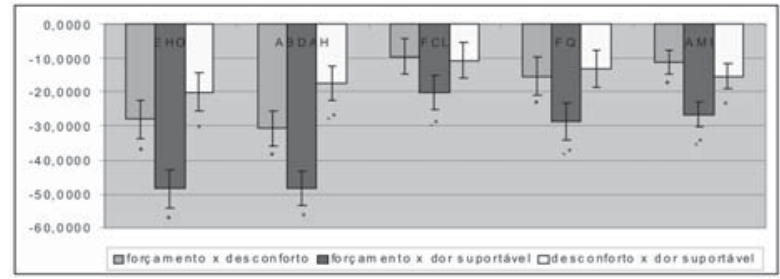

$1^{\circ}$ : extensão horizontal do ombro; $2^{\circ}$ : abdução da articulação do ombro; $3^{\circ}$ : flexão da coluna lombar; $4^{\circ}$ : flexão do quadril; $5^{\circ}$ : abdução dos membros inferiores. ${ }^{*} p<0,05$ a mesma articulação. Para o movimento abdução de membros inferiores (AMI) verifica-se uma correlação no nível correspondente à "mobilidade" 20 .

Estes resultados permitem constatar a existência de correlação significativamente válida entre o arco de movimento articular, a tensão aplicada e a percepção de esforço. Além disso, pode-se perceber uma proporcionalidade existente entre essas medidas, como são apresentadas nos Gráficos 1 e 2.

Pode-se verificar que não ocorreram diferenças significativas apenas nos movimentos de flexão de coluna lombar (FCL), nas comparações das sensações forçamento $X$ desconforto e desconforto $X$ dor suportável. $O$ mesmo ocorre para flexão de quadril (FQ) na comparação da sensação desconforto X dor suportável.

De acordo com o Gráfico 2, pode-se verificar que houve diferença significativa para a sensação forçamento comparada à dor suportável, e para desconforto comparado à dor suportável em todos os movimentos avaliados.

A comparação entre forçamento e desconforto apresentou diferença significativa apenas no movimento de abdução da articulação do ombro (AAO).

\section{DISCUSSÃO}

Os resultados encontrados nesta pesquisa corroboram estudos encontrados na literatura ${ }^{21}$, que descrevem a PSE como um instrumento poderoso na avaliação da

Gráfico 2 - Múltiplas comparações entre as tensões aplicadas nos segmentos corporais, aferidas pelo dinamômetro, e as percepções de esforço, conforme a escala PERFLEX.

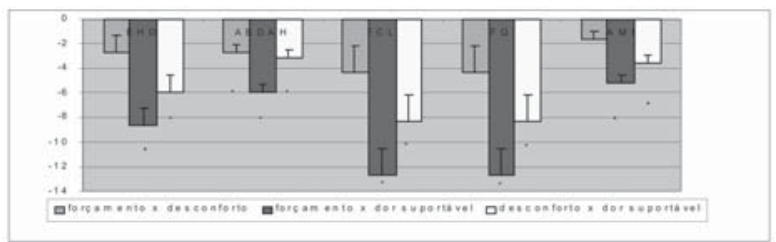

EHO - Extensão Horizontal do Ombro; $A A O$ - Abdução da Articulação do Ombro; FCL - Flexão da Coluna Lombar; FQ - Flexão do Quadril; e AMl - Abdução dos Membros Inferiores. * $p<0,05$ 
flexibilidade, podendo ser alterada pelo treinamento, ao provocar o aumento dos arcos articulares originais.

Em estudos com militares ${ }^{\mathbf{2 1}}$, concluiu-se que indivíduos fisicamente ativos eram capazes de se classificar com precisão, por meio da PSE, em alguns tipos de flexibilidade, o que corrobora os resultados deste estudo, que encontrou correlação significativa entre a PSE e as medidas objetivas em alguns movimentos de flexibilidade.

Outro estudo ${ }^{23}$ encontrou uma correlação positiva entre a categorização estabelecida para os desempenhos de certas habilidades pelos professores e a autocategorização dos mesmos, proposta pelos alunos. Este achado apóia a possibilidade da auto-avaliação por PSE da intensidade necessária para obtenção de arcos de movimentos crescentes, assim como foi apresentado em trabalho ${ }^{\mathbf{1 8}}$ que esclarece um mecanismo conhecido como aumento da capacidade de tolerância à tensão do alongamento, transformando-o em flexionamento.

Em revisão realizada sobre a percepção de desconforto à tensão do alongamento ${ }^{\mathbf{2 4}}$, pode-se perceber que, em qualquer atividade reflexa contrátil, a flexibilidade é predominantemente influenciada pelo componente muscular, em detrimento do componente neural, evidenciando assim que a percepção de esforço exercido será determinante na avaliação da flexibilidade.

Os resultados deste estudo indicam que a Escala de Esforço Percebido na Flexibilidade (PERFLEX) apresenta grande potencial para a avaliação da intensidade necessária, a fim de se atingir os grandes arcos de movimento articular que caracterizam a flexibilidade, conforme demonstram as correlações alcançadas entre os métodos utilizados, principalmente a goniometria.

Após a validação do método, poder-se-á avaliar o esforço despendido na atividade laboral e na cotidiana por diversos profissionais, em especial o grupo que motivou o presente estudo, os enfermeiros.

Ressalte-se que a flexibilidade é uma componente indispensável ao condicionamento físico ${ }^{25}$, e dispor de um instrumento validado com estas características será de grande importância.

A partir dos resultados encontrados nesta pesquisa, recomenda-se: o aumento da amostra, com o objetivo de dar maior significância estatística para a generalização dos resultados; a realização de estudos com outras populações, a fim de verificar o comportamento e a aplicabilidade da escala; e a validação científica da Escala de Esforço Percebido na Flexibilidade - PERFLEX.

\section{REFERÊNCIAS}

1. Dantas EHM, Soares JS. Flexibilidade aplicada ao personal training. Fit Perf J. $2001 ; 1(0): 7-12$.
2. Dantas EHM. Alongamento e flexionamento. $5^{\mathrm{a}}$ ed. Rio de Janeiro: Shape; 2005.

3. Farinatti PTV. Flexibilidade e esporte: uma revisão da literatura. Rev Paul Educ Fís. 2000; 14(1):85-95.

4. Johanne D, Luc N, Line R, Patrick F, Annie R, Chantal V. Validity of the assessment of life habits in older adults. J Rehabil Med. 2004;36:177-82.

5. American College of Sports Medicine. ACSM`s guidelines for exercise testing and prescription. $7^{a}$ ed. Indian: Lippincott Williams \& Wilkins; 2006.

6. Unick J, Kieffer HS, Cheesman W, Feeney A. The effects of static and ballistic stretching on vertical jump performance in trained women. J Strength Cond Res. 2005;19:206-12.

7. Hutton RS. Neuromuscular basis of stretching exercises. In: Komi PV, editor. Stength and power in sport. vol. 3. The encyclopedia of sports medicine. Oxford: Blackwell Scientific; 1992.

8. Magnusson SP, Simonsen EB, Aagaard P, Sørensen H, Kjaer M. A mechanism for altered flexibility in human skeletal muscle. J Physiol. 1996;497(P+1):291-8.

9. Borg G. Psychophysical bases of perceived exertion. Med Sci Sports Exerc. 1982;14(5):377-81.

10. Day ML, McGuigan MR, Brice G, Foster C. Monitoring exercise intensity during resistance training using the session RPE scale. J Strength Cond Res. 2004;18(2):353-8.

11. Unick J, Kieffer HS, Cheesman W, Feeney A. The acute effects of static and ballistic stretching on vertical jump performance in trained women. J Strength Cond Res. 2005;19(1):206-12.

12. Farinatti PTV, Araújo CGS, Vanfraechem JHP. Influence of passive flexibility on the ease for swimming learning in pre-pubescent and pubescent children. Science et Motricité. 1997;31:16-20.

13. De Deyne PG, Hayatsu K, Meyer R, Paley D, Herzenberg JE. Muscle regeneration and fiber type transformation during distraction osteogenesis. J Orthop Res. 1999;17(4):560-70.

14. Alter MJ. Science of flexibility. $3^{a}$ ed. Champaign: Human Kinetics; 2004.

15. Varejão RV, Dantas EMH, Matsudo SMM. Comparação dos efeitos do alongamento e do flexionamento, ambos passivos, sobre os níveis de flexibilidade, capacidade funcional e qualidade de vida do idoso. Rev Bras Ciênc Mov. 2007;15(2): 87-95

16. Organização Mundial de Saúde. Estratégias da OMS sobre a medicina tradicional (2002 - 2005). Genebra: OMS; 2002

17. Dantas EHM, Carvalho JLT, Fonseca RM. O Protocolo LABIFIE de Goniometria. Rev Trein Des. 1997;2(3):21-34.

18. Gajdosik RL. Passive extensibility of skeletal muscle review of the literature with clinical implications. Clin Biomech. 2001;16(2):87-101.

19. Silva EP, Freitas Z, Dantas EHM. Como avaliar a flexibilidade. Fit Perf J. 2004;3(2):61-4

20. Sigmound R. Estatística não-paramétrica. $5^{a}$ ed. São Paulo: McGrawHill; 2004.

21. Mitchell UH, Myrer JW, Hopkins JT, Hunter I, Feland JB, Hilton SC. Acute Stretch Perception Alteration Contributes to the Success of the PNF "Contract-Relax" Stretch. J Sport Rehabil. 2007;16(3):85-92.

22. Knapik JJ, Jones BH, Reynolds KL, Stabb JS. Validity of self-assessed physical fitness. Am J Prev Med. 1992;8(6):367-72

23. Xiang $P$, Lee $A$. The development of selfperceptions of ability and achievement goals and their relations in physical education. Res $Q$ Exerc Sport. 1988;69(3):231-41.

24. Mchungh MP, Kemenic IJ, Fox BM, Glein GW. The role of mechanical and neural restraints to joint range of motion during passive stretch. Med Sci Sports Exerc. 1998; 30(6):928-32

25. ACSM. The recommended quantity and quality of exercise for developing and maintaining cardiorespiratory and muscular fitness, and flexibility in health adults. Med Sci Sports Exerc. 1998;30(6):975-91. 